EPJ Web of Conferences 59, 16003 (2013)

DOI: $10.1051 /$ epjconf/20135916003

(C) Owned by the authors, published by EDP Sciences, 2013

\title{
The energy band structures of the warm dense plasmas with micro-structures inherited from original carbon nanotubes
}

\author{
Q.-L. Dong ${ }^{1,2, a}$, J. Yang ${ }^{2}$, Z.-T. Jiang ${ }^{3}$, Z.-M. Sheng ${ }^{2,4}$ and J. Zhang ${ }^{2,4}$ \\ 1 School of Physics and Optoelectronic Engineering, Ludong University, \\ Yantai 260405, China \\ 2 Beijing National Laboratory for Condensed Matter Physics, Institute of Physics, \\ Chinese Academy of Sciences, 100190 Beijing, China \\ 3 Department of Physics, Beijing Institute of Technology, 100081 Beijing, China \\ ${ }^{4}$ Department of Physics, Shanghai Jiao Tong University, 200240 Shanghai, China
}

\begin{abstract}
The energy band structures of the warm dense plasmas with micro-structures inherited from single-walled carbon nanotubes are studied in detail by using the symmetry-adapted tight-binding model with the electron-ion core Coulomb interactions as the plasma effects. It is found that both the symmetry and the degeneracy of the calculated bands normal for the solid micro-structured targets may be destroyed by the electron-ion core Coulomb interactions. Moreover, the calculated Fermi levels of the micro-structured warm dense plasmas are about $1 \mathrm{eV}$ higher than those of the original carbon nanotubes, while the transition energies of the warm dense plasmas are almost always smaller than those for the original carbon nanotubes.
\end{abstract}

\section{INTRODUCTION}

Warm dense matter (WDM) $[1,2]$ is the state of matter between the condensed and plasma states. As a transient state, WDM has a density of the same order as solid, and a temperature of a few tens of thousands of kelvins (a few electronvolts), and even possibly keeps the original ionic structure unchanged. The ultra-fast laser-target and particle-beam-target experiments are giving quantitative measurements on WDM during the sudden heating of the solid targets [3-6]. With the availability of ultra-short (a few femtoseconds) extreme ultraviolet (XUV) lasers and other ultra-fast x-ray radiation sources, it is possible to ionize the electrons from a large fraction of the atoms at the focal region, producing the warm dense plasmas with the temperature of a few $\mathrm{eVs}$, while keeping the original ionic structure unchanged because the ions' moving time scale is larger than a few picoseconds.

In the present paper, we adopt the common physical variable transition energies to characterize the energy band structures of the warm dense plasmas produced from single-walled carbon nanotubes (SWCNTs). By taking into consideration the Coulomb interaction between the free electrons and the not-yet-moved ion cores (referred as e-i Coulomb interaction hereafter.), the plasmas effects are investigated by using the symmetry-adapted nonorthogonal tight-binding model (SA-NTB) [7, 8]. The calculated results show sharp changes in the energy band structures of the warm dense plasmas in comparison with the original carbon nanotubes.

\footnotetext{
ae-mail: qldong@aphy.iphy.ac.cn
}

This is an Open Access article distributed under the terms of the Creative Commons Attribution License 2.0, which permits unrestricted use, distribution, and reproduction in any medium, provided the original work is properly cited. 

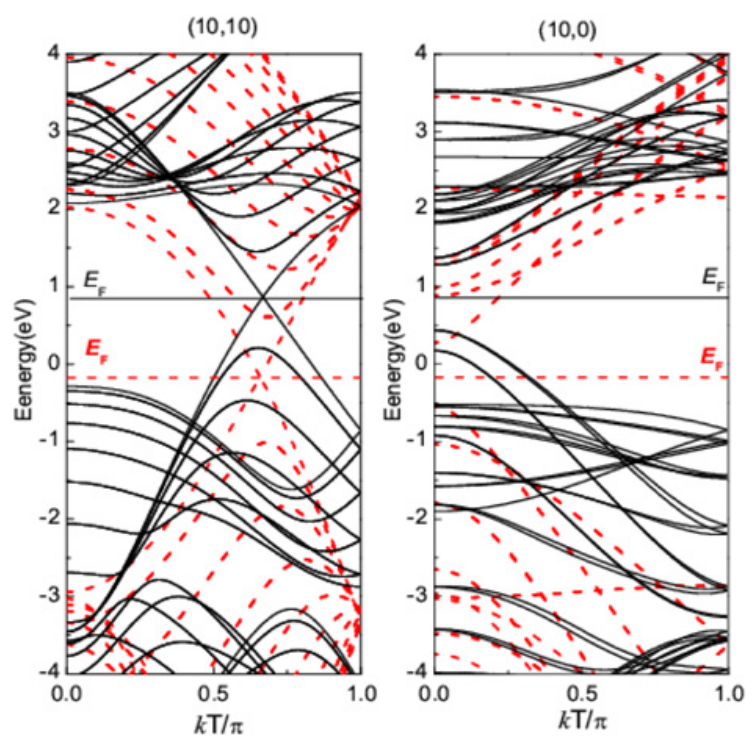

Figure 1. The energy band structure of carbon nanotubes $(10,10)$ and $(10,0)$ in the energy range $(-4,4) \mathrm{eV}$. The solid lines (black) represent the SA-NTB results of the warm dense SWCNTs and the dashed lines (red) represent the SA-NTB results of cold solid SWCNTs. The horizontal lines $\left(E_{\mathrm{F}}\right)$ in the figure are the Fermi level.

\section{RESULTS AND DISCUSSION}

Both the hybridization of the $\sigma-\pi$ states and the overlap of the different atomic orbitals are considered in the SA-NTB model. The hamiltonian matrix elements and the overlap matrix elements are calculated for all atomic pairs in the range between 1 and 7 bohrs.

\subsection{Energy band for the nanotube warm dense plasmas}

As it is well known, the carbon tubes may be metallic or semiconducting. Therefore, as an example here we select the typical armchair tube $(10,10)$ and the zigzag tube $(10,0)$, which corresponds to the metallic and semiconducting states of tubes, respectively. First of all, in the left panel of Figure 1, we show the calculated band structures of the tube $(10,10)$ obtained on the basis of the SA-NTB model. The results of the SWCNTs warm dense plasmas and the original solid tubes are represented by the solid lines (black) and the dashed lines (red), respectively. Obviously, the SA-NTB band structure of these SWCNTs warm dense plasmas deviates greatly from that of original tubes. In the case of the armchair tube $(10,10)$, though the crossings of the lowest conducting band and the highest valence band corresponding to the Fermi levels $E_{\mathrm{F}}$ appear at the same wave vector of $k=2 \pi / 3$, the Fermi level of the warm dense tube is about $1 \mathrm{eV}$ higher than that of the original tube. This indicates that the Fermi level may be lifted up with the plasma effects introduced by considering the e-i Coulomb interaction. Furthermore, the bottoms of the other conducting bands, as well as the tops of the other valence bands, calculated in the presence of the plasmas effects of the e-i Coulomb interaction are localized far away from the Brillouin zone boundary of $k=\pi$, relative to the case without the plasmas effects denoted by the dashed lines. Moreover, the energy level degeneracy of original tubes at the Brillouin zone boundary is wholly removed and some new kinds of degeneracy appear. This demonstrates that the e-i Coulomb attraction interaction in the SWCNTs warm dense plasmas can lower the symmetry of the band structures with respect to the Fermi level and destroy the degeneracy of the band structures at $k=\pi$. 

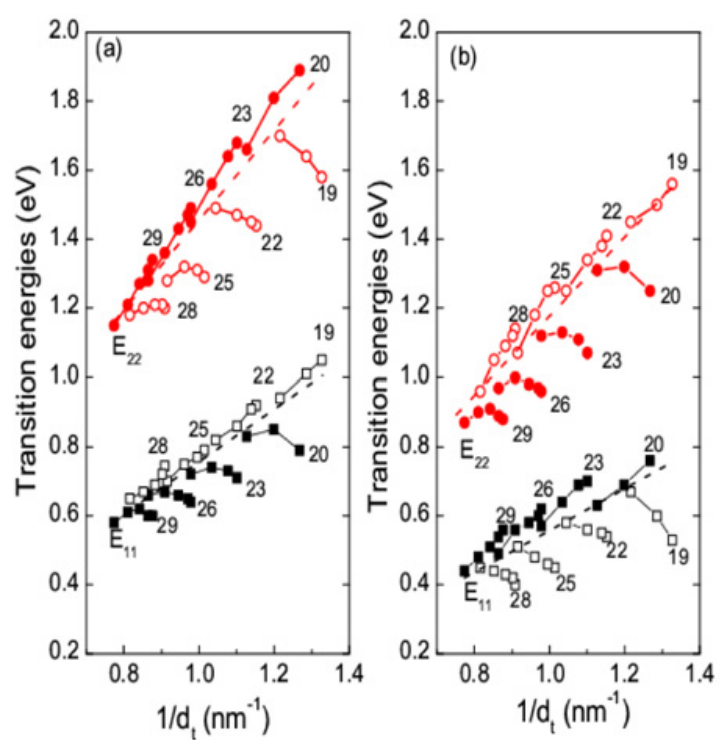

Figure 2. (a) The optical transition energy $E_{11}$ and $E_{22}$ of different warm dense SWCNTs calculated by SA-NTB model versus their inverse diameters of $1 / d_{\mathrm{t}}$, where the open and filled symbols denote the Mod 1 and Mod 2 SWCNTs, and the black squares and red circles correspond to the $E_{11}$ and $E_{22}$ states, respectively. (b) is the same as (a), but the transitions $E_{11}$ and $E_{22}$ is from SA-NTB model for the cold SWCNTs. The integers indicate the $2 \mathrm{n}+\mathrm{m}$ family indices and the black (red) dash lines are linear fit for $E_{11} \propto 1 / \mathrm{d}_{t}\left(\right.$ or $\left.E_{22} \propto 1 / \mathrm{d}_{t}\right)$. Within a branch, the indices of two neighboring nanotubes are related by $\left(n^{\prime}, m^{\prime}\right)=(n-1, m+2)$. For example, when $2 \mathrm{n}+\mathrm{m}=20$, the nanotube indices are $(10,0),(9,2),(8,4)$ and $(7,6)$, respectively, with their diameters increased.

For comparison, the energy-band structure of the zigzag tube $(10,0)$ is shown in the right panel of Figure 1. Obviously a gap between the conducting band and the valence one is observed, clearly showing the semiconducting property of the tube. Just as the armchair tube $(10,10)$, the calculated Fermi level of the warm dense tube $(10,0)$ is also about $1 \mathrm{eV}$ higher than that of the original tube $(10,0)$. The transition energy $E_{11}=0.84 \mathrm{eV}$ of the warm dense tube is a little higher than that $E_{11}=0.77 \mathrm{eV}$ of the original tube, while the transition energy $E_{22}=1.25 \mathrm{eV}$ of the warm dense tube is much lower than that $E_{22}=1.89 \mathrm{eV}$ of the original tube because the second lowest conduction-band state is pulled down and the second highest valance band states is pushed up. It is demonstrated that the symmetries of the energy bands for both the $(10,10)$ and $(10,0)$ tubes are significantly lowered on account of the different hybridization of the $\sigma-\pi$ states induced by the curvature on the different $\mathrm{C}-\mathrm{C}$ bond.

\subsection{Transition energies for the warm dense plasmas from the original semiconducting carbon nanotubes}

In the following, we turn to investigate the transition energies which can be used to characterize the optical excitations in the SWCNTs by using the SA-NTB model proved by Popov [7, 8]. The transition energies $E_{11}$ and $E_{22}$ of 32 different SWCNTs warm dense plasmas (left panel) and original solid SWCNTs (right panel) with their diameters in the range of $0.75-1.32 \mathrm{~nm}$ are obtained as plotted in Figure 2 . The integer numbers in the figures are used to denote the $2 n+m$ family numbers, that is to say, each number of $2 n+m$ represents one family. It can be found that the transition energies $E_{11}$ and $E_{22}$ closely depend on the SWCNTs' diameters and chiral angles, as well as the values of $v=\operatorname{Mod}(2 n+m$, 3). There exist similar behaviors between Figures 2(a) and (b) which are summarized as follows: (1) The transition energies $E_{11}$ and $E_{22}$ increase with the increasing of the inverse diameter $1 / d_{\mathrm{t}}$, which could be approximately described by a linear function of $E_{i i} \propto 1 / d_{\mathrm{t}}$. (2) Within the same $2 n+m$ family, the 
maximal deviation from the linear fit of $1 / d_{\mathrm{t}}$ occurs for the zigzag-like tubes, and the minimal deviation for the close-to-armchair tubes. This family behavior can be understood according to the wrapping effect and the tube's curvature effect.

On the other hand, the curves in Figure 2 show sharp differences. The first is that the transition energies of SWCNTs show different deviations from the linear fit line of $E_{i i} \propto 1 / \mathrm{dt}$. For $E_{11}$ transitions, the $v=1(\operatorname{Mod} 1)$ branches bend upwards from the linear fit line, while the $v=2(\operatorname{Mod} 2)$ branches downwards. In contrast, the situation for $E_{22}$ transitions is different, where the Mod 1 branches bend downwards and the Mod 2 branches upwards. However, in Figure 2(b), for $E_{11}$ transitions, the $v=1(\operatorname{Mod} 1)$ branches bend downwards from the linear fit line, while the $v=2(\operatorname{Mod} 2)$ branches upwards. Also, the situation for $E_{22}$ transitions is that the Mod 1 branches bend upwards and the Mod 2 branches downwards. Under the influence of the e-i Coulomb interaction in the SWCNTs warm dense plasmas, the $E_{i i}$ property dependence on the chirality for the Mod 1 SWCNTs seems to be contrary to that for the Mod 2 SWCNTs. The second is that the transition energies $E_{i i}^{(a)}$ are almost larger than the corresponding $E_{i i}^{(b)}$ for the same SWCNT, where $E_{i i}^{(a)}$ and $E_{i i}^{(b)}$ correspond to the data in Figure 2(a) and (b), respectively. This behavior can be understood well by the plasmas effects.

\section{SUMMARY}

Using the SA-NTB model supplemented by the e-i Coulomb interaction as the plasma effect, we investigate the transition energies $E_{i i}$ of the different semiconducting SWCNTs warm dense plasmas. It is shown from our calculations that the e-i Coulomb interaction or the plasma effects on the transition energies depend on the original SWCNT's diameter and chirality. Within the same $2 n+m$ family, the transition differences $\left(E_{i i}\right)$ caused by the plasma effects on the higher energy branch increase with decreasing the chiral angle, while those on the lower energy branch decrease with decreasing the chiral angle. The Fermi level of the warm dense matter/plasma is also found to be lifted up about $1 \mathrm{eV}$ compared to the cold SWCNTs.

This work is jointly supported by National Natural Science Foundation of China (Grant Nos 11074297, 11274152 and 10974015), National Basic Research Program of China (973 Program) (Grant No. 2007CB815101), and Program for New Century Excellent Talents in University (Grant No. NCET-08-0044). Also, we thank Professor V. N. Popov for constructive suggestions.

\section{References}

[1] S. Mazevet, M. P. Desjarlais, L. A. Collins, J. D. Kress, N. H. Magee, Phys. Rev. B 71, 016409 (2005)

[2] D.O. Gericke, K. Wunsch, A. Grinenko, J. Vorberger, Journal of Physics: Conference Series 220, 012001 (2010)

[3] R. Ernstorfer, M. Harb, C. T. Hebeisen, G. Sciaini, T. Dartigalongue, R. J. Dwayne Miller, Science, 323, 1033 (2009)

[4] S. Ku, S. Son, Sung Joon Moon, Phys. Plasmas 17, 052702 (2010)

[5] O. Peyrusse, J. Phys.: Condens. Matter 20, 195211 (2008)

[6] M. W. C. Dharma-wardana, Phys. Rev. E 73, 036401 (2006)

[7] V. N. Popov, New J. Phys. 6, 17 (2004)

[8] V. N. Popov, L. Henrard, Phys. Rev. B 70, 115407 (2004)

[9] Z. M. Li, V. N. Popov, Z. K. Tang, Solid State Communitions 130, 657 (2004) 